\title{
ROLE OF MRI IN EVALUATION OF FEMALE PELVIC MASSES IN COMPARISON TO ULTRASONOGRAPHY
}

\author{
Tushar Prabha', Sunny Goyal², Hemant Kumar Mishra³, Ankur Aggarwal ${ }^{4}$
}

\section{HOW TO CITE THIS ARTICLE:}

Tushar Prabha, Sunny Goyal, Hemant Kumar Mishra, Ankur Aggarwal. "Role of MRI in Evaluation of Female Pelvic Masses in Comparison to Ultrasonography". Journal of Evolution of Medical and Dental Sciences 2014; Vol. 3, Issue 59, November 06; Page: 13328-13334, DOI: 10.14260/jemds/2014/3775

ABSTRACT: The study was conducted in 50 female patients having clinically suspected Pelvic masses at physical examination underwent both USG and MRI. Sonographic assessment of uterus, bilateral adnexal regions and pouch of Douglas was done in cases of suspected masses. Other organs such as liver and peritonium were also visualized in cases of malignancies for metastatic lesions. MRI assessment of lesions was done within a short interval of 1-2 days. Most common lesions evaluated on imaging were benign uterine lesions (15 cases, 34.09\%), benign adnexal lesions(13 cases, 29.54\%), Malignant adnexal (10 cases, 22.73\%), Malignant utero cervical lesions ( 6 cases, 13.64\%), 2 cases had indeterminate type lesions, 2 lesions proved to be normal bowel loops on MRI and in 2 cases MRI could not be performed. Cystic lesions were commonly seen in adenexa (15 out 21) while solid lesions were common in utero-cervical region (18 out 23).Out of these 14 were complex cystic adenexal masses and 10 were malignant. Uterocervical lesions were carcinoma cervix. In cases of larger lesions MRI was the best. Both TVS and trans-abdominal USG were less informative in comparison to MRI. Two cases of dermoid were diagnosed confidently on the MRI because of superior soft tissue contrast and fat detection. Whereas the diagnosis was in doubt on USG. By MRI better assessment with improved imaging capability was possible in invasion of surrounding structure in 10 cases, lymphadenopathy in 3 cases, ascites in 13 cases, peritoneal implant in 6 cases, encasement in 1 case and distant metastases in 1 case. Ascites and metastases were diagnosed in all the patients on USG. Results indicated higher diagnostic capability of MRI for dermoid, hemorrhagic cyst and pre-treatment assessment of pelvic malignancies; also it is problem solving tool in cases of indeterminate pelvic masses on USG. MRI is relatively safe, non-operator dependent, has high signal noise ratio and it is an excellent modality for staging pelvic malignancies, it has superior soft tissue contrast and large field of view. All these features offer a distinct advantage of MRI over USG in assessment of female pelvic masses.

INTRODUCTION: MRI has become an important modality in the evaluation of female pelvic lesions; USG remains the examination of choice for initial assessment of patients with suspected benign gynaecological disease, given its relative safety and lower cost.(1,2) USG may be limited by technical considerations (e.g. operator dependence, patient's body habitus, low signal noise ratio and is inadequate in staging pelvic malignancies).(1,3-5) MRI is safe because of no radiation exposure as is in the USG; while CT has a danger of -radiation exposure. MRI is considered the next step in the imaging assessment of benign disease of the uterus and adnexa and is becoming the primary imaging modality for evaluating gynaecologic malignancies. $(6,7)$

The multi-planer imaging capability of MRI, superior soft tissue contrast and large field of view offer distinct advantages over both USG and CT (Computer Tomography) in the assessment of gynaecologic abnormalities. Although ultrasonography remains the initial modality used in 
evaluating clinically suspected gynecologic disease.(1,2,3) MRI can offer supplemental diagnostic information in cases of suboptimal or equivocal ultrasound examination, and in patients in whom there is discrepancy between sonographic findings and physical examinations.Endo-vaginal sonography provide improved spatial resolution. USG is not as useful as CT and MRI in the detection of the loco-regional extent of pelvic cancer.

Generally uterine masses are mainly solid as opposed to ovarian masses which are mainly cystic. If the mass can be shown to arise from the uterus, it is usually a benign leiomyoma. Leiomyomas are common differential diagnosis of solid adnexal mass. Frequently fibroids can be diagnosed sonographically by their decreased echogenecity and decreased sound through transmission, even though the relationship to the uterus is obscured. Occasionally it may be impossible to determine the exact origin of the mass by the sonography than MRI may be helpful. A tailored MRI may be used to help clarify and confirm cases that are not as definitive sonographically.

On MRI fibroids may 'also present variable appearances. They are usually low signal such that pedunculated ones can mimic feces. Fortunately ovarian cancer, the important differential diagnosis, never appears similar. However, degenerated fibroids can have moderately high signal intensity on T2w scans. There may even be a peculiar nodular appearance to massive myomas that can mimic an ovarian malignancy. The definitive diagnosis can be made by showing the stretching of myometrium around the base of lesion. This, the most definite sign, is much better appreciated on MRI than sonography.

Patient Preparation and Method of Examination: Trans-abdominal and selected trans-vaginal examinations were performed to evaluate the pelvic masses. TAS was done with full bladder and curvilinear probe of medium (5MHz) frequency was used. The full urinary bladder was required to provide an acoustic window and to displace the bowel loops. Transverse axial and sagittal scanning plane images were obtained through the short and long axis of uterus.

Trans-vaginal probe insonate at higher frequency i.e. $7-9 \mathrm{MHz}$ was used with consequent improved spatial resolution over the lower frequency TAS probes. The probe was covered with a protective sheath (a condom) and adequate coupling gel was applied, with the patient in the lithotomy position after having emptied her bladder. The sagittal and semi coronal planes were imaged.

For MRI, patients were imaged with an empty bladder after fasting for at least 4 hours. Imaging was performed with the patient supine. A phased array coil was used in most cases.

In both T1W \& T2W MRI in axial, sagittal, coronal planes were evaluated. T1W images offer excellent contrast between the pelvic organs and adjacent fat allow optimal detection of lymph nodes and are necessary for tissue and fluid characterization (essential for hemorrhagic and fat containing lesions). T2W images sequences are needed to demonstrate the zonal anatomy of the uterus and vagina and to facilitate the identification of normal ovaries. In addition $\mathrm{T} 2 \mathrm{~W}$ images are usually superior in depicting pathologic conditions of uterus and ovaries.

Additional MRI imaging included selective application of fat saturation imaging. It was done to differentiating fat, blood and proteinaceous fluid in hyper intense lesions in T1W images.

For pelvic MRI axial, sagittal and coronal fast spin echo T1 weighted images were obtained by using TE-minimal full, TR used was approx. 560ms, echo train length was 3, band width was approx. 2.83 in most of the cases. 
Slice thickness was $5 \mathrm{~mm}$, spacing approx. $1 \mathrm{~mm}$. Frequency, phase and NEX was adjusted according to the image quality. Frequency direction in axial, sagittal images is anterior/ posterior while in coronal images it is superior/ inferior. Patient is kept supine in feet first position.

Images were obtained in 2D mode, coil used was TORSOPA.

In T2 weighted fast spin echo images TE and TR is high in this study TE used was 102 and TR was approx. 4200, echo train length was 12 , band width was 15.6 , slice thickness, spacing, patient's position, mode of imaging and coil remained the same as that of T1 weighted images.

In fat saturated images the protocol was same as that of T2 weighted sequences except for the fat suppression option was selected.

Patient was imaged by USG first than MRI (performed within a short interval of 1-2days to avoid any change in the mass that might occur as a result of cyclical bleeding) to look for the various features; which form the mainstay of the differential diagnosis of the pelvic masses. These include shape, size, margins, internal architecture (cystic/ solid), organ of origin, extent of involvement of surrounding structures, absence or extent of metastatic involvement and presence of ascites or peritoneal implant. These features on complete assessment can help to make a diagnosis or at least narrow down the differential diagnosis.

Information collected was tabulated in master chart to analyze observations and then statistical analysis of the data on the basis of history, ultra sonographic, MRI findings was done. Cases were followed up; histopathological diagnosis was considered final and was obtained by biopsy or FNAC of mass. Follow up sonography was done in cases managed conservatively by drugs or by radiotherapy or in cases of spontaneous resolution. The USG and MRI findings were compared.

\begin{tabular}{|c|c|c|c|c|c|c|c|}
\hline $\begin{array}{c}\text { Age group } \\
\text { (In Yrs) }\end{array}$ & \multicolumn{3}{|c|}{ USG (n=50) } & \multicolumn{4}{c|}{ MRI (n=48) } \\
\cline { 2 - 8 } & Cystic & Solid & Complex & Cystic & Solid & Complex & Others \\
\hline \multirow{2}{*}{35} & 0 & 10 & 2 & 3 & 4 & 3 & 1 \\
& $(0.00)$ & $(20.00)$ & $(4.00)$ & $(6.25)$ & $(8.33)$ & $(6.25)$ & $(2.08)$ \\
\hline \multirow{2}{*}{$35-44$} & 0 & 8 & 6 & 4 & 6 & 4 & 0 \\
& $(0.00)$ & $(16.00)$ & $(12.00)$ & $(8.33)$ & $(12.50)$ & $(8.33)$ & $(0.00)$ \\
\hline \multirow{2}{*}{$>45$} & 0 & 15 & 9 & 6 & 8 & 8 & 1 \\
& $(0.00)$ & $(30.00)$ & $(18.00)$ & $(12.50)$ & $(16.67)$ & $(16.67)$ & $(2.08)$ \\
\hline \multirow{2}{*}{ Total } & $\mathbf{0}$ & $\mathbf{3 3}$ & $\mathbf{1 7}$ & $\mathbf{1 3}$ & $\mathbf{1 8}$ & $\mathbf{1 5}$ & $\mathbf{2}$ \\
& $\mathbf{( 0 . 0 0 )}$ & $\mathbf{( 6 6 . 0 0 )}$ & $\mathbf{( 4 2 . 0 0 )}$ & $\mathbf{( 2 7 . 0 8 )}$ & $\mathbf{( 3 7 . 5 0 )}$ & $\mathbf{( 3 1 . 2 5 )}$ & $\mathbf{( 4 . 1 6 )}$ \\
\hline
\end{tabular}

Table 1: Comparison of pelvic masses according to internal consistency on USG and MRI

Note: MRI could not be done in 2 patients

\begin{tabular}{|c|c|c|c|c|c|c|}
\hline \multirow{2}{*}{ Pelvic mass } & \multicolumn{3}{|c|}{ USG } & \multicolumn{3}{c|}{ MRI } \\
\cline { 2 - 7 } & Benign & Malignant & Total & Benign & Malignant & Total \\
\hline \multirow{2}{*}{$<5$} & 13 & 3 & 16 & 11 & 3 & 14 \\
& $(26.00)$ & $(6.00)$ & $(32.00)$ & $(22.92)$ & $(6.25)$ & $(29.17)$ \\
\hline \multirow{2}{*}{$>5$} & 21 & 13 & 34 & 21 & 13 & 34 \\
& $(42.00)$ & $(26.00)$ & $(68.00)$ & $(43.75)$ & $(27.08)$ & $(70.83)$ \\
\hline \multirow{2}{*}{ Total } & $\mathbf{3 4}$ & $\mathbf{1 6}$ & $\mathbf{5 0}$ & $\mathbf{3 2}$ & $\mathbf{1 6}$ & $\mathbf{4 8}$ \\
& $\mathbf{( 6 8 . 0 0 )}$ & $\mathbf{( 3 2 . 0 0 )}$ & $\mathbf{( 1 0 0 . 0 0 )}$ & $\mathbf{( 6 6 . 6 7 )}$ & $\mathbf{( 3 3 . 3 3 )}$ & $\mathbf{( 1 0 0 . 0 0 )}$ \\
\hline
\end{tabular}

Table 2: Distribution of Adnexal masses according to size on USG and MRI 
DISCUSSION: Regarding the accuracy of USG and MRI for the diagnosis of pelvic masses, on USG correct evaluation of site of origin and morphology was possible in $42 \%$ cases while on MRI it was possible in $93.75 \%$ cases.

On both imaging modalities all malignant adnexal masses were more than $5 \mathrm{~cm}$ in size, 10 out of 13 benign adnexal masses were more than $5 \mathrm{~cm}$ in size which is contrary to the study done by Meire et al (1978). In his study he found that cysts $<5 \mathrm{~cm}$ with thin septa and no solid nodules were generally benign.

Regarding the accuracy of USG and MRI for the diagnosis of pelvic masses, on USG correct evaluation of site of origin and morphology was possible in $42 \%$ cases while on MRI it was possible in 93.75\% cases. This difference was found to be significant ( $\mathrm{P}$ value is $<0.001$ ). MRI provided new information in $48 \%$ cases. This information is related to the origin of mass, spread of tumour and internal consistency (such as fat or haemorrhagic contents within the lesion) in most of the cases. By MRI, presence of fat was detected in $4 \%$ cases which were heteroechoic on USG and could not be diagnosed correctly.

All these masses were proved to be dermoid on histopathology. Heterogenous lesions without fat contents were seen in $24 \%$ cases and were due to tiny haemorrhagic foci. These findings correlate very well with Jain KA Jeffery RB JR (1994) who concluded that MRI is superior in detection and differentiation of haemorrhagic masses from the dermoids (fat containing masses) when fat suppression technique was used. Comparable was what Scoutte LM McCarty SM et al (1994) found that MRI is $100 \%$ sensitive and $99 \%$ specific in prospective diagnosis of dermoid.

Both MRI and TVS superbly demonstrated the internal architecture of haemorrhagic cyst as multiple fine septations thereby allowing a specific diagnosis. Although MRI allowed identification of blood on the basis of characteristic signal intensity on T1 and T2 weighted images and contributed to a diagnosis of haemorrhagic mass, identification of a focal haemorrhagic mass does not allow a specific diagnosis of haemorrhagic cyst. MRI also helped in staging of the malignant lesions which involve the determination of encasement of vessels. It was detected in $2 \%$ cases; invasion was detected in $20 \%$ cases, pelvic lymphadenopathy in $6 \%$, peritoneal implants in $12 \%$, ascites in $26 \%$, and distant metastasis in $2 \%$ cases.

In $42 \%$ cases MRI provided confirmation of the suspected lesions on USG with improved images while only $6 \%$ cases were diagnosed confidently on USG.

CONCLUSION: Pelvic organs and surrounding structures were thoroughly evaluated by USG and MRI. We concluded that:

1. Pelvic masses are more common above the age of 45 years.

2. Multiparity associated with high incidence of pelvic masses.

3. Most of the malignant pelvic masses are seen in multipara above the age of 45 years.

4. Postmenopausal bleeding has a strong association with the carcinoma cervix.

5. Adnexal masses are usually cystic while the uterine masses are solid in texture.

6. As the complexity and size of the cystic adnexal masses increases there are increased chances of malignancy.

7. Small pelvic masses are better assessed by the TVS while in cases of large pelvic masses MRI give better detail of mass and surrounding structure.

8. Fat containing masses such as dermoids are correctly diagnosed by MRI. 


\section{CASE STUDY}

9. Hemorrhagic cyst or intralesional hemorrhages are confidently detected on MRI.

10. MRI is better than USG in determining the origin of female pelvic mass. It is also more accurate in classifying the adnexal masses into benign and malignant.

11. In cases of pre-treatment assessment of malignancies, invasion of surrounding structure, encasement, invasion of vessels or assessment of lymphadenopathy, peritoneal implant, ascites and distant metastases are better appreciated by MRI.

12. Staging in cases of malignancy is possible by MRI.

Results indicated higher diagnostic capability of MRI for dermoid, hemorrhagic cyst and pretreatment assessment of pelvic malignancies; also it is problem solving tool in cases of indeterminate pelvic masses on USG.

MRI is relatively safe, non-operator dependent, has high signal noise ratio and it is an excellent modality for staging pelvic malignancies, it has superior soft tissue contrast and large field of view. All these features offer a distinct advantage over USG in assessment of female pelvic masses.

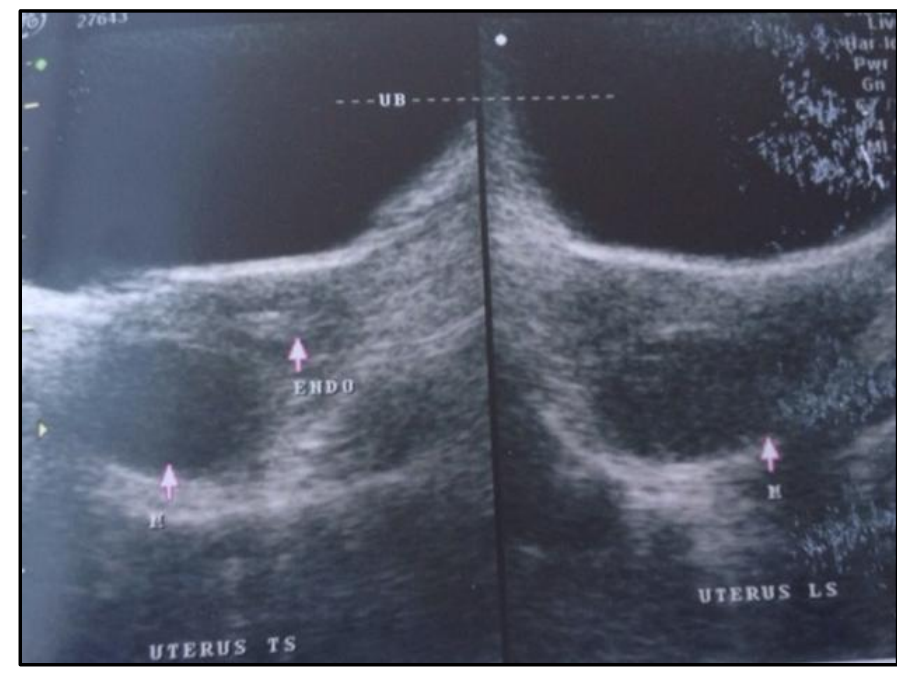

\section{USG SHOWING CYSTIC MASS IN RIGHT ADENEXA}

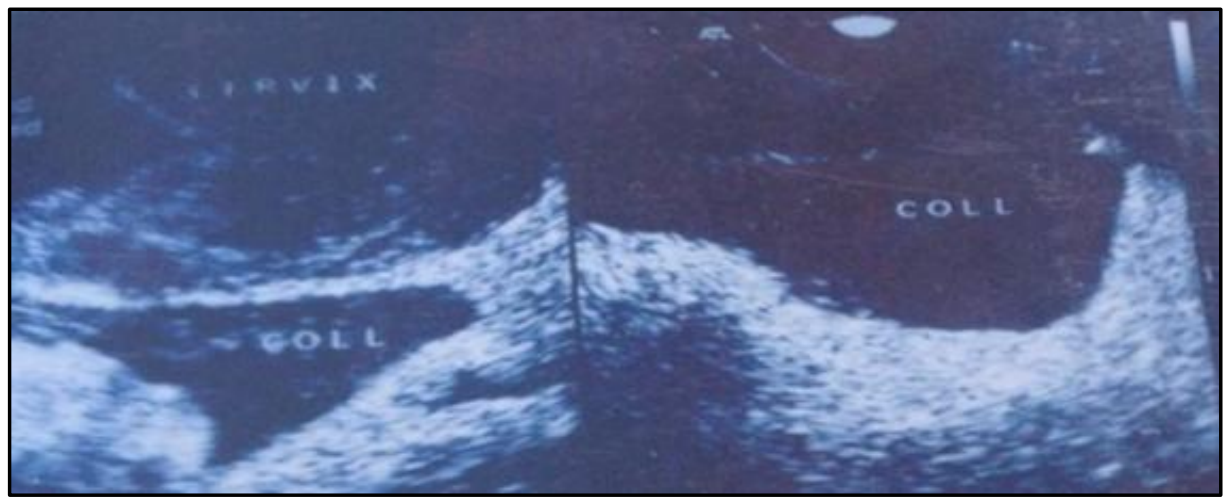

USG SHOWING HYPO-ECHOIC COLLECTION IN POD 


\section{CASE STUDY}

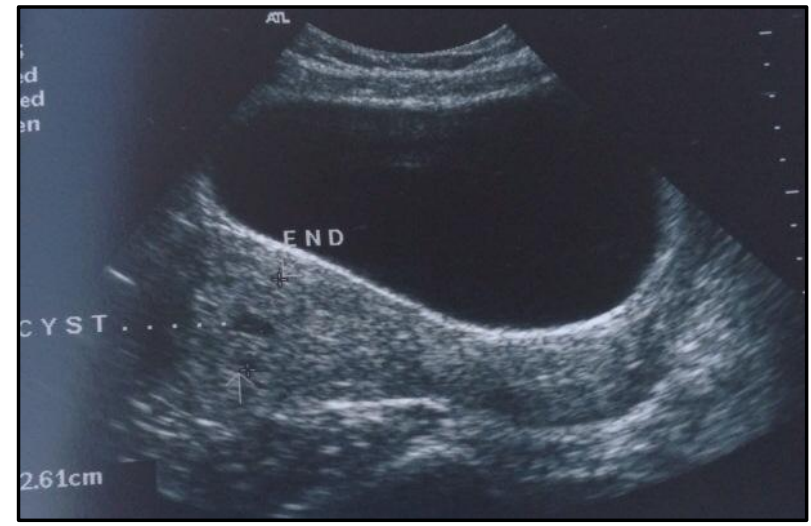

USG SHOWING CYSTIC LESION IN ENDOMETRIAL CAVITY

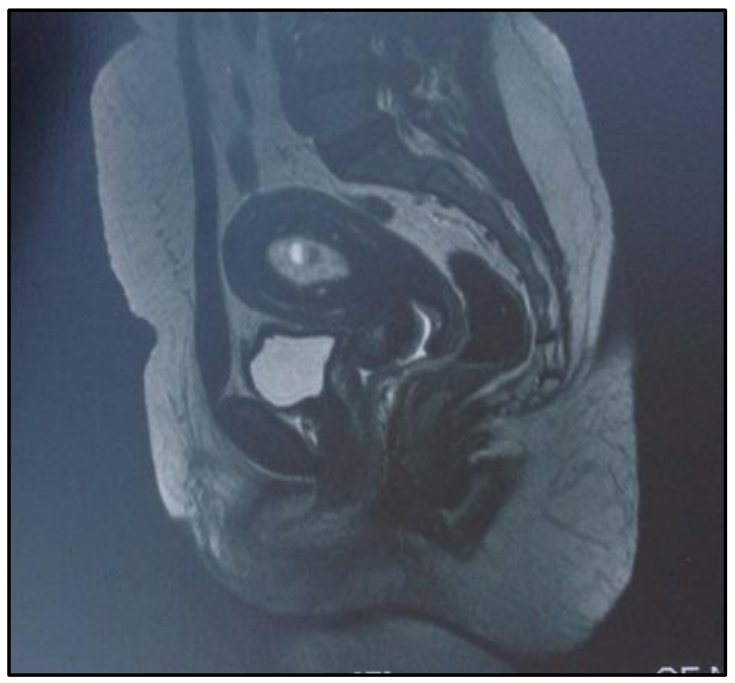

T2W M.R.I. showing endometrial cystic lesion with soft tissue component

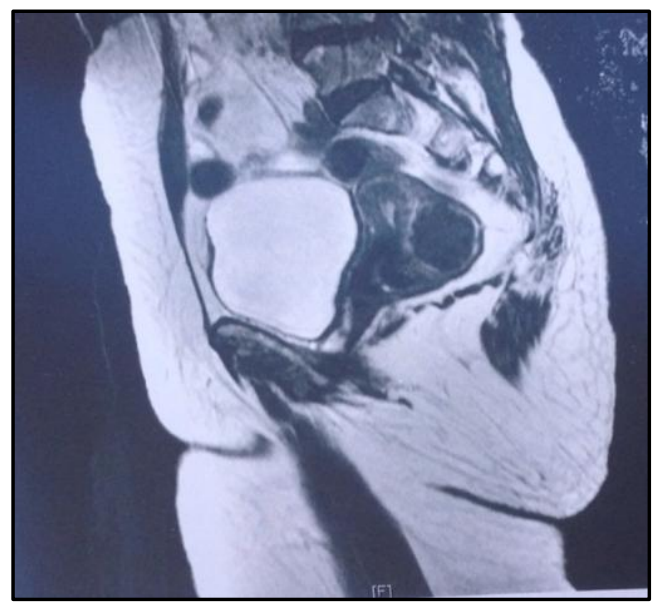

T2W M.R.I. showing subserosal fibroid in posterior wall of uterus 


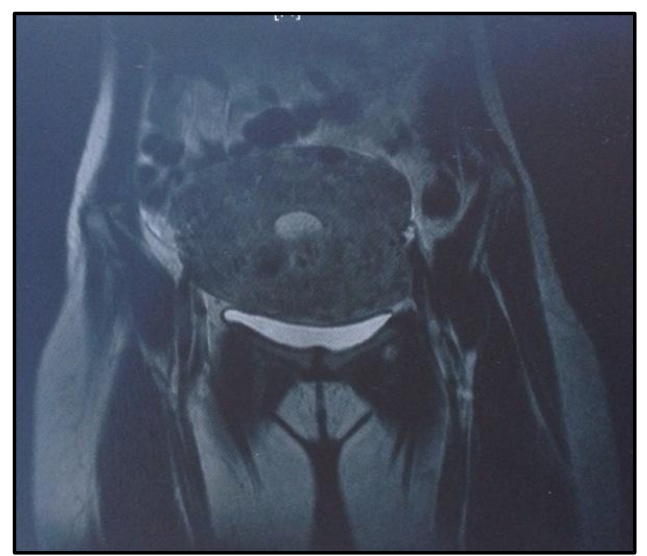

\section{T2W CORONAL IMAGE SHOWING MULTIPLE HYPOINTENSE FOCI IN MYOMETRIUM -MULTIPLE LEIOMYOMAS}

\section{REFERENCES:}

1. Andreottic RF, Zusmer NR, Sheldon JJ et al. Ultrasound and magnetic resonance imaging of pelvic masses. Surg. Gynecol Obster 166:327, 1988.

2. Buy JN. Pelvic endometriosis. J Radiol, 2001 Dec; 82 (12 Pt 2): 1867-79.

3. Carol M. Runack, Diagnostic Ultrasound 3rd ed. 527-579.

4. Charles B Higgns, Hedvig Hricak, Clyde A. Helms. Magnetic Resonance Imaging of the body III edition p.3-24.

5. Curtis M, Hopkins MP, Zarlingo T et al. Magnetic resonance imaging to avoid laparotomy in pregnancy. Obstet Gynaecol. 1993:82 (5): 833-6.

6. Grainger and Allisons, Diagnostic Radiology A textbook of Medical Imaging Edition- 4, 22012222. 2004.

7. GRAY"s Anatomy. The Anatomical Basis of Clinical practice, 39th ed. 2005.

\section{AUTHORS: \\ 1. Tushar Prabha \\ 2. Sunny Goyal \\ 3. Hemant Kumar Mishra \\ 4. Ankur Aggarwal}

\section{PARTICULARS OF CONTRIBUTORS:}

1. Senior Resident, Department of Radiodiagnosis, Mahatma Gandhi Medical College and Hospital, Jaipur.

2. P. G. Resident, Department of Radiodiagnosis, Mahatma Gandhi Medical College and Hospital, Jaipur.

3. Professor and HOD, Department of Radiodiagnosis, Mahatma Gandhi Medical College and Hospital, Jaipur.
4. P. G. Resident, Department of Radiodiagnosis, Mahatma Gandhi Medical College and Hospital, Jaipur.

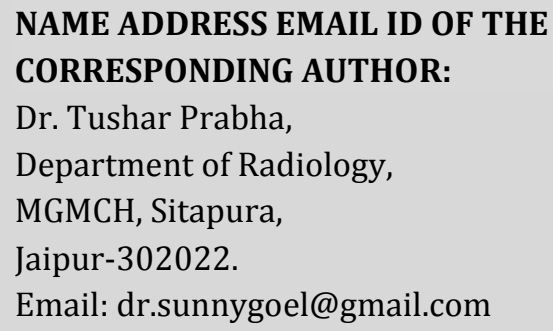

Date of Submission: 15/10/2014.

Date of Peer Review: 16/10/2014.

Date of Acceptance: 05/11/2014.

Date of Publishing: 06/11/2014. 